\title{
Über die energetische Fläehenregel.
}

\author{
Von \\ Prof. M. Pfaundler, München. \\ Mit 1 Textabbildung. \\ (Eingegangen am 18. Februar 1921.)
}

Wie bekannt ist die Annahme verbreitet, daß der Energieumsatz (E) verschiedener Tiere in der Zeiteinheit unter sonst gleichen Bedingungen der Körperoberfläche proportional sei. Diese Lehre geht auf Karl Berg mann zurück, der im Jahre 1847 ausführte: Durch jede Körperoberflächeneinheit bei Mensch und Tier gehe physikalisch notwendig in der Zeiteinheit eine bestimmte Wärmemenge verloren. Die Konstanz der Körpertemperatur bei Homoiothermen fordere andauernd entsprechende Nachschaffung von Wärme, es bestimme also ceteris paribus die Kör peroberfläche den wärmeerzeugenden Stoffwechsel.

Teils in gleichem, teils in ähnlichem Sinne bewegten sich - nunmehr gestützt auf die grundlegenden Versuche von Regnault und Reiset (1849) - die Ausführungen mehrerer Nachfolger, so jener von Ramea $x^{1}$ ) 1857, Immermann 1865, Liebermeister 1875, Richet

1) Was die Arbeit von Ramea ux betrifft, die sich auf die Forschungen von Lavoisier, Despretz und Dulong und Gavarret stützt, ist hier einiges anzuführen, weil sich darüber in der Literatur Irrtümer eingeschlichen haben. Einmal sei bemerkt, daß man die Arbeit in den „Mémoires de I'Académie Royale de Belgique", wie es im Zitat heißt, vergeblich sucht, und zwar sowohl in deren Classe des lettres etc. als auch in der Classe des sciences, sowohl in der Quartwie in der Oktavausgabe; vielmehr ist sie abgedruckt in den davon unabhängig ausgegebenen „Mémoires couronnés et Mémoires des savents étrangers publ. par l'Académie Royale de Belgique“, Bd. 29, Brüssel 1858. Der Titel heißt: „Des lois suivant lesquelles les dimensions du corps, dans certaines classes d'animaux déterminent la capacité et les mouvements fonctionelles des poumons et du coeur". Präsentiert im Mai 1857. Ferner ist festzuhalten, daß Ra mea ux nach seiner Angabe im Verein mit Sarrus der genannten Akademie bereits im Jahre 1838 einige Betrachtungen über dieses Thema vorgelegt hat. Diese Betrachtungen sind ungedruckt geblieben und es geht aus der 20 Jahre später erschienenen Publikation von Rameaux nicht hervor, welche Thesen oder Gedankengänge aus letzterer etwa schon früher gefaßt worden sind. Bei solcher Sachlage kann Rameaux keinesfalls eine Priorität gegenüber Karl Berg mann eingeräumt werden, dessen 1847 erschienenes Buch ihm übrigens ganz unbekannt geblieben zu sein scheint Endlich ist zu konstatieren, daß bei Ra mea ux - entgegen späteren Angaben - 
1885 und insbesondere von Rub ner seit 1883, welch letzterer die Lehre nach verschiedenen Richtungen erweiterte und zu ihrer Stütze ein erhebliches Zahlenmaterial beibrachte.

Seither wurden aber nicht allein diese Zahlen von verschiedenen Seiten angefochten, sondern es wurden auch die theoretischen Grundlagen der Bergmannschen und der ursprünglichen Rubnerschen Lehre als unhaltbar erkannt, es wurde auch gezeigt, daß eine Proportionalität zwischen Energieumsatz und Hautoberfläche - soferne oder insoweit sie wirklich bestünde - nicht die Abhängigkeit des Energieumsatzes von dieser Hautoberfläche (die überdies objektiv gar nicht begrenzbar und meßbar ist, Verf. 1916) beweist, sondern bestenfalls die Proportionalität zwischen Energieumsatz und einer beliebigen ideellen Körperfläche.

Welche solche Flächen außer der Haut- oder äußeren Körperoberfläche noch in Betracht kommen, wurde verschiedentlich erwogen. Nach Weis mann sollen Le uckart und Herbert S pencer schon vor

von einer Proportionalität zwischen dem Energieumsatz und dem Werte $P^{\frac{2}{3}}$ nirgends die Rede ist, daß er sich überhaupt weder direkt auf besagten Umsatz, noch auf die Körpermasse bezieht, sondern auf die eingeatmete Luft und ihr Sauerstoffvolumen einerseits, auf die Körperflächen oder zweiten Potenzen von linearen Dimensionen andererseits. Die Grundlagen des Autors sind folgende physikalische, geometrische bzw. physiologische Gesetze: 1. Ähnliche Körper gleichen Materials verlieren in der Zeiteinheit Wärmemengen, die ihren ,Oberflächen proportional sind. 2. Die Volumina ähnlicher Körper sind proportional den dritten Potenzen, die Flächen den zweiten Potenzen ihrer homologen linearen Dimensionen. 3. Die von den Tieren erzeugte Wärme rührt von Verbrennungen her, bewirkt durch die von den respiratorischen Oberflächen aufgenommenen Sauerstoffmengen. Geometrisch ähnliche Tiere gleicher Art, so schließt Rameaux, verbrauchen Sauerstoffmengen und atmen in der Zeiteinheit Luftvolumina ein, die den Quadraten ihrer homologen Strecken proportional sind. Weitere, und, wie er selbst zugibt, hypothetische Voraussetzungen lassen ihn zu folgender Haupt. these gelangen: Für Wirbeltiere mit Lungenatmung und konstanter Körpertemperatur (Säugetiere und Vögel) bestimmen die Dimensionen des Tieres die Kapazität seiner Respirationsorgane und die Zahl der Inspirationen, ferner die Kapazität des Herzens und die Zahl der Herzschläge dergestalt, daß, wenn diese Größen von einem Individuum mit gegebenen Dimensionen bekannt, sind, ihr Wert für ein anderes Individuum derselben Art durch Rechnung ermittelt werden kann, soferne man nur dessen homologe Dimensionen kennt; und zwar ist:

$$
n^{\prime}=n \cdot \sqrt{\frac{d}{d^{\prime}}} \quad \text { und } \quad v^{\prime}=v \frac{d^{\prime 2}}{d^{2}} \cdot \sqrt{\frac{d^{\prime}}{d}},
$$

wobei $n$ bzw. $n^{\prime}$ die Anzahl der Atemzüge oder Pulsschläge in der Zeiteinheit, $d$ bzw. $d^{\prime}$ eine der Körperstrecken, $v$ bzw. $v^{\prime}$ die Kapazität der Lunge oder des Herzens bedeuten. Daraus kann man unter der Voraussetzung, daß Herz- und Lungenkapazität der Gesamtkörpermasse proportional seien, ableiten $P=$ Konst. $\times d^{*}$, was von dem sonst angenommenen $P=$ Konst. $\times d^{2}$ abweichen und nicht zum Satze $E=$ Konst. $\times P^{2}$ führen würde, den man dem Autor irrtümlich zuschreibt. 
ca. 60 Jahren an die sogenannten ernährenden Flächen gedacht haben; von Hösslin führte dies näher aus und Mühlmañn kam darauf wieder zurück; Liebermeister und manche andere dachten auch an verschiedene andere innere Körperoberflächen, z. B. an die Respirationsfläche.

Meissner 1857 zeigte, auf Karl von Vierordt gestützt, daß die vermeinte Proportionalität auch ganz andere Gründe haben bzw. andere Erklärung finden könnte, die sich nicht auf irgendwelche Oberflächenleistungen, sondern auf Kreislaufverhältnisse bezieht. In seiner 1888 erschienenen Schrift behandelte dann H. v. Hösslin das Problem mit einer weder vorher noch nachher erreichten Umsicht und Gründlichkeit. Das Ergebnis war der von Rub ner unterdessen zum Gesetz erhobenen Lehre nicht günstig.

Jüngst versuchte ich den Stand der Frage dahin zu präzisieren, daß der Energieumsatz höchstwahrscheinlich mit der Hautoberfläche als solcher nichts zu tun habe, daß er aber vielleicht eine Kör perflächenf u k ktion allgemein sei, wobei die Frage offen bleiben müsse, ob es sich um die oder jene, ob es sich überhaupt um eine bestim m te Fläche im Körper handelt. In diesem Sinne spricht man vielleicht zweckmäßig nicht von einem energetischen Oberflächengesetz, sondern von einer energetischen Fächenregel.

Jeder Körper weist eindimensionale, zweidimensionale und dreidimensionale Größen auf, mit anderen Worten Strecken (Längen, Breiten und Höhen), Flächen und, Volumina, für welch letztere man im allgemeinen bei tierischen Körpern ohne große Fehler zu machen auch die entsprechenden Massen oder Gewichte setzen kann. Einem stereometrischen Gesetze zufolge ist bei ähnlichen Körpern 'jede Fläche $(F)$ proportional der zweiten Potenz jeder Strecke $(L)$, ferner proportional der $2 / 3$-Potenz jeder Massendimension, beispielsweise des Körpergewichtes $(P)$, endlich proportional jedem Quotienten zwischen einer Masse und einer Strecke (also beispielsweise auch dem sog. Zentimetergewichte). Arithmetisch ausgedrückt gilt also folgendes :

$$
\left.F=f_{1} L^{2}=f_{2} \cdot P^{2}=f_{3} \cdot P L^{-1 *}\right)
$$

Die energetische Flächenregel wird man hiernach so fassen können:

$$
\left.E=\varphi \cdot F=\varphi_{1} L^{2}=\varphi_{2} P^{\frac{2}{3}}=\varphi_{3} \cdot P L^{-1 * *}\right) .
$$

Dies gilt wie gesagt für ,ähnliche Körper", d. h. (bei Tieren) für gleichbleibende Statur. Bei veränderlicher Statur (nach Art oder nach

*) $f_{1}, f_{2}, f_{3}$ numerische Faktoren. $f_{2}$ ist, wenn $F$ die äußere Körperoberfläche von Tieren bedeutet, der sog. Meeh-Vierordtsche Koeffizient.

**) $\varphi$ konstanter Energieumsatz der Flächeneinheit unter bestimmten Bedingungen. $\varphi_{1}=\varphi f_{1}, \varphi_{2}=\dot{\varphi} f_{2}, \varphi_{3}=\varphi f_{3}$. 
Alter) müssen die besagten Proportionalitäten der geometrischen Größen untereinander aufhören und man möchte meinen, es wäre leicht zu ermitteln, ob für eine bestimmte und für welche Fläche die Proportionalität mit dem Energieumsatz erhalten bleibt; diese Fläche wäre gegebenenfalles mit dem Energieumsatz als in kausaler Beziehung stehend oder für ihn bestimmend anzusehen. Eine Möglichkeit, die Frage auf diesem Wege zu entscheiden, hat sich bisher nicht ergeben, weil der Meeh-Vierordt sche Koeffjzjent ein im Verhältnis zu den Fehlergrenzen der Umsatzbestimmung zu wenig empfindlicher Staturindex ist, bzw. weil man Fälle von gröblich abweichendem Meeh-VierordtKoeffizienten (als welohe ich die Handflügler vorgeschlagen habe) bisher zu Energieumsatzbestimmungen nicht herangezogen hat. Es soll der Versuch gemacht werden, hier auf anderen Wegen einen kleinen Schritt weiter zu kommen.

Überblickt man die gesamte Ontogenese eines Säugers, so gelangt man zum Ergebnis, daß der Übergang vom intrauterinen zum extrauterinen Dasein trotz der eingreifenden Umgestaltung vieler Lebensbedingungen an den Wachstums- und. Entwicklungsvorgängen so gut wie nichts ändert; was auch Friedenthal, Rössle u. a. jüngst mit Nachdruck betont haben. Dies läßt annehmen, daß die für. Wachstum und Entwicklung maßgeblichen, also auch die energetischen Gesetze vor und nach der Geburt im wesentlichen dieselben sind. Dann müßte auch für den Fötus der Energieumsatz Körperflächen proportional sein, woraus jedenfalls zu ersehen wäre, daß die sich jenseits der Geburt an den „Ernährungsflächen“ abspielenden Verdauungs- und Resorptionsprozesse damit nichts zu tun haben. Aber auch nach anderer Richtung wirft diese Erwägung Licht. Anfangs dachte man sich die Beziehungen zwischen der Körpero erfläche und dem Energieumsatz vermittelt durch die auf die Haut einwirkenden Kältereize, nämlich begründet in der ,chemischen Temperaturregulierung". Später mußte man nicht allein solche Wirkungsweise auf thermische Reize überhaupt erweitern, sondern auch zugeben, daß ebenso gut mechanische, aktinische und chemische Reize auf die äußeren oder die inneren freien Oberflächen dabei im Spiele sein können. Für den Embryo und Fötus bleiben aber von all diesen Reizen fast nur gewisse chemische, nämlich vorwiegend assimilatorisch wirkende übrig, deren Angriffspunkt zunächst in der Oberfläche der Körperzellen vermutet werden muß. Hiernach entsteht die Frage, wie sich die Gesamtsumme der Oberflächen der Körperzellen im Laufe des Wachstums etwa verhält, was sich natürlich nur unter Annahme gewisser vereinfachender Bedingungen feststellen läßt, beispielsweise unter der Annahme, die Zellen seien der einzige Körperbestandteil, seien gleichgroß, kugelförmig und von der Dichte 1 (Abb. Reihe A). Wenn ihr 
Halbmesser jeweils $r$ beträgt und ihre Zahl jeweils $N$, so ist die Summe ihrer Oberflächen $\Sigma_{0}=N \cdot 4 \pi r^{2}$, das Körpergewicht aber

woraus sich berechnet:

$$
P=N \cdot \frac{4 \pi}{3} r^{3},
$$

$$
\Sigma_{0}=p^{\frac{2}{3}} \cdot N^{\frac{1}{3}} \cdot f_{1}
$$

oder, wenn der Energieumsatz der Gesamtzelloberfläche proportional ist :

$$
E=P^{\text {号}} \cdot N^{\frac{1}{3}} \cdot f_{2} .
$$

Hiernach würde der Energieumsatz also nicht einfach mit der Größe $P^{\frac{2}{3}}$ wachsen, sondern überdies mit der auf Eindrittelpotenz reduziertén Zahl der den Körper zusammensetzenden Zellen. Dies würde der energetischen Flächenregel nur dann völlig entsprechen, wenn das Wachstum durch reine „Hypertrophie", d. h. Vergrößerung der einzelnen Zellen zustande käme, nicht aber gleichzeitig auch durch „Hyperplasie“, Zellvermehrung. Man weiß, daß es Tiere gibt, bei denen dies von einer
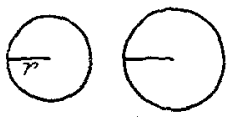

Abb. 1. (Reihe A). Zunahme der Zelleg an ZahI und Größe.
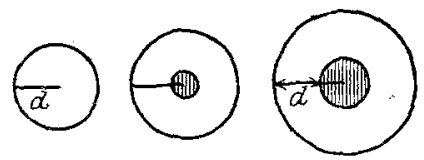

Abb. 1. (Reihe B). Ebenso unter Bildung eines paraplasmatischen Kernes.

gewissen Entwicklungsstufe an durchaus der Fall ist. Ob diese Tiere (Vermes usw.) auf ihren Energiewechsel untersucht sind, kann ich nicht angeben. $\mathrm{Da} \mathrm{B}$ er grundlegende Besonderheiten darbiete, ist nicht eben wahrscheinlich. Beim Säugetier ist reine Zellvergrößerung als wachśtumsbestimmendes Koment jedenfalls auf gewisse Entwicklungsstufen und gewisse Gewebsgruppen beschränkt, wie beispielsweise auf die Ganglienzellen und großenteils auch auf die Mroskelzellen im extrauterinen Leben.

Das Ergebnis legt nahe einen dem Faktor $N^{\frac{1}{2}}$ in obiger Gleichung entgegenwirkenden, einen den Energieumsatz sukzessive mindernden Faktor im Laufe des Wachstums zu suchen. Diesen hat schon Kassowitz in der fortschreitenden Bildung von ,reizfesten" Bestandteilen, sog. Metaplasma vermutet. Neuerdings spricht Friedenthal in gleichem Sinne vom Paraplasma, worunter er insbesonders die Summe von Differenzierungsprodukten, die ,Fibrillenmaschine“ des Körpers versteht und das sich in stets zunehmendem Maße an Stelle von Protoplasma setze. Letzteres allein will der Autor als lebende Substanz im engsten Sinne des Wortes aufgefaßt wissen und ihm allein schreibt er Teilungsfähigkeit zu. Auch wenn die Mehrzahl der Histologen mit der 
gegenteiligen Ansicht recht haben sollte, darf doch wohl angenommen werden, daß der Stoffwechsel im reizfesten Teil der Körpermasse träger ist, so daß mit der fortschreitenden Paraplasmierung, dem relativen Rückgange des Protoplasmas, der Energieumsatz pro Körpergewichtseinheit abnimmt.

Solche Gedankenrichtung scheint von der Flächenfunktionslehre gänzlich abzulenken. $\mathrm{Ob}$ aber nicht doch Beziehungen zwischen dem Kassowitz-Friedenthalschen Faktor und dem Bergmann-Rubnerschen bestehen, ist der Prüfung wert.

Stellt man sich den Gesamtkörper (Masse $P$ ) als eine ursprünglich nur aus Protoplasma (Masse Pr) bestehende Kugel vor, deren Durchmesser im Laufe des Wachstums allmählich zunimmt, während von ihrem Zentrum aus ein gleichfalls wachsender kugliger Kern von Paraplasma $(P a)$ entsteht (siehe Abb., Reihe B), um den sich eine Kugelschale aus Protoplasma von der Wandstärke $d$ legt, so errechnet sich einfach folgende Beziehung :

$$
\operatorname{Pr}=d f_{1} P^{\frac{2}{3}}-d^{2} f_{2} P^{\frac{1}{3}}+d^{3} f_{3},
$$

mit anderen Worten: die jeweilige lebende Masse im engern Sinne des Wortes steht in einer (freilich nicht reinen) proportionalen Beziehung zur Zweidrittelpotenz des Körpergewichtes, soferne der Wert $d$ konstant bleibt.

Die Vorstellung eines massiven paraplasmatischen Kernes im Gesamtkörper entfernt sich aber allzusehr von den tatsächliehen Verhältnissen. Weit eher annehmbar erseheint die Vorstellung, daß solches Geschehen, wie hinsichtlich des Gesamtkörpers angenommen, in jedem Elementarteil, in jeder einzelnen Zelle ablaufe. Auf dieser Basis gelangt man zum Ergebnis:

$$
P r=d f_{1} \cdot N^{\frac{2}{3}} \cdot P^{\frac{2}{3}}-d^{2} f_{2} \cdot N^{\frac{2}{3}} \cdot P^{\frac{1}{3}}+d^{3} f_{3} \cdot N^{\frac{3}{3}} .
$$

Ähnliche Formeln ergeben sich auch bei gewissen anderen Annahmen über die Topographie des Paraplasmas- oder Fibrillensystemes in und um die Zellen.

Obige Formel läßt. sich unter der Bedingung, daß $N^{\frac{1}{2}} \cdot d$ konstant sei, reduzieren auf:

$$
\operatorname{Pr}=K \cdot f_{1} \cdot P^{\frac{2}{3}}-K^{2} f_{2} \cdot P^{\frac{1}{3}}+K^{3} \cdot f_{3}(K, \text { numerische Konstante }) .
$$

d. h. eine (unreine) Proportion zwischen der Protoplasmamasse und dem Werte $P^{2}$ bleibt dann bestehen, wenn jederzeit $N^{\frac{1}{3}}=\frac{K}{d}$ oder mit anderen Worten: wenn die Zahl der Körperzellen (reduziert a uf $1 / 3$ Potenz) beim Wachstum in gleichem Maße steigt, wie die Stärke der Protoplasmaschicht der einzelnen Zellen abnimmt. Vermeinte Reziprozität biologisch zu begründen erscheint nicht aussichtslos. 
Immerhin wird man finden, daß die Reduktion der energetischen Flächenregel auf die Paraplasmierung so keineswegs zwanglos gelingt - zumal wenn man berücksichtigt, daß sicher auch das Paraplasma an den Stoffwechselvorgängen teilnimmt, daß es in hundertfältig verschiedener Form und in ungleicher Menge und Beschaffenheit in und zwischen die Zellen ausgeschieden wird und daß viele andere Momente einen ziffernmäßigen Überblick über diese Verhältnisse behindern. Gleichwohl wird man sich nicht ohne weiteres entschließen, die nach Rubners Originalzahlen im ganzen leidlich erscheinende Deckung der $P^{\frac{2}{3}}$ - mit der Umsatzkurve des Wachsenden auf bloße Zufälligkeiten zurückzuführen. Vielleicht darf an folgendes erinnert werden.

Nach Bütschli besteht das Protoplasma im wesèntlichen aus einer schaum- oder wabenartig strukturierten Grundmasse (mit gewissen geformten und ungeformten Einschlüsšen, welch letztere aber nicht die eigentlichen Träger der Lebensvorgänge sind). Hiernach wäre die lebende Substanz also in keiner Form ein Massensystem, sondern stets ein Flächensystem. Ein solches Flächensystem müßte eine Gesamtoberfläche von f. $P^{\frac{2}{s}}$ haben. Dann wäre die energetische Flächenregel allerdings recht durchsichtig - aber doch nur mit der Beschränkung, daß die einzelnen Teile der Gesamtkörpermasse in gleichem oder doch wenigstens in proportional bleibendem Maße während der ganzen Entwicklungszeit an den Stoffwechselvorgängen teilnehmen. Dies ist sehr unwahrscheinlich. Die minder aktiven Teile vermehren sich fortwährend, nicht allein absolut, sondern auch relativ. Ein Ausdruck dessen ist vielleicht das Absinken der Ümsatzkurve unter die $P^{\frac{1}{3}}$-Kurve im Alter, wie sie nach einer Reihe von Beobachtern sicher statthat. Da die Paraplasmabildung aber offenbar schon im Beginn der Entwicklung einsetzt, bleibt hier eine Unklarheit bestehen, die erst auf Grund weiteren, und zwar zuverlässigen und wirklich vergleichbaren Materiales ïber den Energieumsatz verschieden großèr und verschieden alter Tiere zu beseitigen möglich sein wird.

Zusammenfassung. Das Absinken des Energieumsatzes pro Masseneinheit im Laufe der Entwicklung hat man hauptsächlich auf zweierlei Weise zu erklären versucht: einerseits durch die Annahme, es handle sich beim Energieumsatz nicht um eine Massen-, sondern um eine Flächenfunktion, anderseits durch die Annahme, es werde die lebende tätige Masse im engeren Sinne des Wortes, das Protoplasma, in fortschreitendem Maße durch eine im Stoffwechsel minder aktive Masse, das Paraplasma, ersetzt.

Zưr Flächenfunktionslehre wird im Vorstehenden ausgeführt, daß unter gewissen Voraussetzungen das Bestimmende weder die äußere Haut- noch die eine oder andere innere Schleimhautoberfläche sein könne, sondern eher die gesamte Assimilationsfläche, das ist die Summe 
der Oberflächen aller am Stoffwechsel teilnehmeniden Elemente und zwar nach Maßgabe dieser Teilnahme - weiter, daß die Struktur des Protoplasmas in diesem, mithin im Organismus überhaupt ein Flächensystem erblicken läßt.

Zur Paraplasmalehre wird gezeigt, daß - wieder unter gewissen und zwar biologisch begründbaren Voraussetzungen - die fortschreitende Paraplasmierung den Umsatz der Gewichtseinheit in solchem Grade reduzieren muß, daß er als eine (allerdings unreine) Körperflächenfunktion erscheinen kann.

\section{Literaturverzeichnis.}

Liebermeister, Handbuch der Pathologie und Therapie des Fiebers. Verlag F. C. W. Vogel 1875. - Rameaux, s. oben. - Zimmermann, Dtsch. Klinik 1865, Nr. 4. - Berg manñ, Über die Verhältnisse der Wärmeökonomie der Tiere zu ihrer Größe. Göttingen 1848, Vandenhoek u. Ruprecht. - v. HöBli n, Über die Ursache der scheinbaren Abhängigkeit des Umsatzes von der Größe der Körperoberfläche. Arch. f. Anat. u. Physiol. Physiol. Abt. 1888. - Weis mann, Über die Dauer des Lebens. Jena 1882, Verl. Fischer. - Mühl mann, Über die Ursache des Alterns. Wiesbaden 1900, Verl. Bergmann. - Pfa undler, Körpermaßstudien an Kindern. Berlin 1906, Verlag Springer. - Friedenthal, Über Wachstum. Ergebn. d. inn. Med. u. Kinderheilk. 8. 1912. - RöBle, Wachstum und Altern. Ergebn. d. allg. Pathol. usw. 18. 1917. - Kassowitz, Die Ursachen des größeren Stoffverbrauches im Kindesalter. Jahrb. f. Kinderheilk. 67. 1908. Allgemeine Biologie. Wien 1899. Vierordt, Meißner. Zitiert nach Hößlin. 